\title{
Improved Measurements of ICRF Antenna Input Impedance at ASDEX Upgrade during ICRF Coupling Studies
}

\author{
I. Stepanov ${ }^{\mathrm{a}, \mathrm{b}}$, J.-M. Noterdaeme ${ }^{\mathrm{a}, \mathrm{b}}$, V. Bobkov ${ }^{\mathrm{a}}$, D. Coster ${ }^{\mathrm{a}}$, H. Faugel ${ }^{\mathrm{a}}$, \\ R. Bilato ${ }^{\mathrm{a}}$, M. Brambilla ${ }^{\mathrm{a}}$, W. Suttrop ${ }^{\mathrm{a}}$, A. Kallenbach ${ }^{\mathrm{a}}$, J. Schweinzer ${ }^{\mathrm{a}}$, \\ E. Wolfrum ${ }^{\mathrm{a}}$, R. Fischer ${ }^{\mathrm{a}}$, A. Mlynek ${ }^{\mathrm{a}}$, V. Nikolaeva ${ }^{\mathrm{c}, \mathrm{d}}$, L. Guimarais ${ }^{\mathrm{c}}$, \\ D. Milanesio ${ }^{\mathrm{e}}$ and the ASDEX Upgrade Team ${ }^{\mathrm{a}}$
}

\author{
${ }^{a}$ Max Planck Institute for Plasma Physics, EURATOM Association, 85748 Garching, Germany \\ ${ }^{b}$ Department of Applied Physics, Gent University, 9000 Gent, Belgium \\ ${ }^{c}$ Universidade Técnica de Lisboa, EURATOM Association/IST, 1049-001 Lisboa, Portugal \\ ${ }^{d}$ Universität Stuttgart, D-70569 Stuttgart, Germany \\ ${ }^{e}$ Politecnico di Torino, 10129 Torino, Italy
}

\begin{abstract}
A new set of diagnostics has been implemented on ASDEX Upgrade to measure the input impedance of the ICRF antennas, in the form of a voltage and current probe pair installed on each feeding line of every antenna. Besides allowing the measurement of the reflection coefficient $\Gamma$ of each antenna port, the probes have two advantages: first, they are located close to the antenna ports $(\sim 3 \mathrm{~m})$ and thus the measurements are not affected by the uncertainties due to the transmission and matching network; second, they are independent of matching conditions.

These diagnostics have been used to study the behavior of the ASDEX Upgrade antennas while changing the plasma shape (low to high triangularity) and applying magnetic perturbations (MPs) via saddle coils. Scans in the separatrix position $R_{\text {sep }}$ were also performed. Upper triangularity $\delta_{\mathrm{o}}$ was increased from 0.1 to 0.3 (with the lower triangularity $\delta_{\mathrm{u}}$ kept roughly constant at 0.45 ) and significant decreases in $|\Gamma|$ (up to $~ 30 \%$, markedly improving antenna coupling) and moderate changes in phase (up to $\sim 5^{\circ}$ ) of $\Gamma$ on each feeding line were observed approximately at $\delta_{\mathrm{o}} \geq 0.29$. During MPs (in $\sim 0.5 \mathrm{~s}$ pulses with a coil current of $1 \mathrm{kA}$ ), a smaller response was observed: $6 \%-7 \%$ in $|\Gamma|$, with changes in phase of $\sim 5^{\circ}$ apparently due to $R_{\text {sep }}$ scans only. As $|\Gamma|$ is usually in the range $0.8-0.9$, this still leads to a significant increase in possible coupled power. Numerical simulations of the antenna behavior were carried out using the FELICE code; the simulation results are in qualitative agreement with experimental measurements. The results presented here complement the studies on the influence of gas injection and MPs on the ICRF antenna performance presented in [4].
\end{abstract}

Keywords: ICRF, coupling, antenna impedance.

PACS: 52.50.Qt

\section{INTRODUCTION}

The well-known sensitivity of ICRF power coupling to the plasma density profile in front of the antenna, due to the fast wave (FW) evanescence layer, naturally leads to a search for ways to modify said density profile to improve coupling. This is especially relevant in view of the large antenna - separatrix gap projected for ITER. One method to improve coupling is local gas injection, which has been extensively studied on JET, Tore Supra, DIII-D and ASDEX Upgrade [1,4]. Two alternative methods are 1) to change the plasma shape (i.e. triangularity $\delta$ ) and 2) to apply magnetic perturbation (MP) fields via saddle coils. The first method is motivated by a previous study of the antenna loading resistance $R_{\mathrm{L}}$ in L-mode discharges at ASDEX Upgrade [2], whereby it was observed that $R_{\mathrm{L}}$ was significantly higher at higher $\delta$. The use of MPs for ELM suppression, on the other hand, is well known to cause changes in the density profile [3]; in addition, changes in $R_{\mathrm{L}}$ during MPs had already been observed on ASDEX Upgrade in non-dedicated discharges prior to this study. Furthermore, new diagnostics installed on the ICRF system have enabled highly detailed measurements of the antenna impedance during these discharges, allowing observation of the behavior of each antenna strap independently. 


\section{ICRF SYSTEM AND DIAGNOSTICS ON ASDEX UPGRADE}

The ICRF system on ASDEX Upgrade consists of 4 two-strap antennas, each strap fed by its own $25 \Omega$ line. These are then joined at a T-section into a single $50 \Omega$ line, where a matching network consisting of two parallel stubs is located (see Fig. 1). The two typical operating frequencies are 30 and $36.5 \mathrm{MHz}$, corresponding to a fundamental hydrogen $(\mathrm{H})$ resonance on the plasma axis at 2 and $2.5 \mathrm{~T}$, respectively.

The main power and input impedance $\left(Z_{\text {inp }}\right)$ diagnostic for each antenna is a pair of directional couplers (DCs) located on the generator side of the matching network (on the matched line section). Although generally reliable, due to finite directivity $(\sim 25$ dB) the DCs can have high measurement errors at high VSWR, e.g., when the antenna $Z_{\text {inp }}$ is out of the matching region. Furthermore, the DCs provide a measurement of the $Z_{\text {inp }}$ of the antenna and transmission and matching networks together, treating the whole as a single-port system. To measure the individual $Z_{\text {inp }}$ at each antenna port, one can use either voltage probe arrays or voltage/current probe pairs located close to each strap feeder (the probe locations are also shown schematically

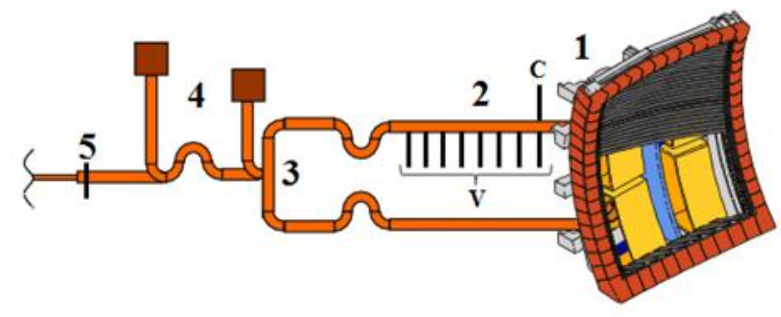

FIGURE 1. One of four ASDEX Upgrade antennas with transmission lines and matching network. 1 - antenna; 2 location of voltage $(\mathrm{V})$ and current $(\mathrm{C})$ probes; 3 - Tsection; 4-matching network; 5 - location of directional couplers.

in Fig. 1). The voltage probe arrays have been shown to have - at present - insufficient accuracy for the desired measurements [6]; the voltage/current probe pairs, in contrast, are sufficiently accurate and have been recently recalibrated and re-installed on each $25 \Omega$ feeding line of all four antennas. The signals are read by logarithmic RF detectors which can measure voltages and currents up to $19 \mathrm{kV}$ and 420 A respectively; the details of the data acquisition system are given in [7].

\section{EXPERIMENTAL SETUP AND MAIN RESULTS}

\section{Effect of Plasma Shape}

Six discharges were dedicated to this study; two at toroidal magnetic field $B_{0}=$ $2.5 \mathrm{~T}$ (on axis) and four at $B_{0}=2 \mathrm{~T}$ (of which three were terminated early); the scenario was heating an $\mathrm{H}$ minority in $\mathrm{D}$ (as well as in the MP studies) and coupled power was between 3.1 and 3.6 MW with all four antennas powered simultaneously between 1.5 and $7 \mathrm{~s}$. During the flat-top phase, central density was $n_{\mathrm{e} 0} \approx 6-$ $7 \times 10^{19} \mathrm{~m}^{-3}$. While the lower triangularity $\delta_{\mathrm{u}}$ was maintained at 0.45 , the upper triangularity $\delta_{\mathrm{o}}$ was increased from 0.1 to $\sim 0.32$. At $\delta_{0}=0.12,0.22$ and 0.32 the separatrix position $R_{\text {sep }}$ was moved from 5 to $7-8 \mathrm{~cm}$ away from the antenna limiter. Plasma density was measured by DCN interferometer (core), Li beam emission spectroscopy and broadband reflectometry (edge); the first two were used to reconstruct a full density profile.

Fig. 2 shows the relevant time traces for discharge 29823, typical for the series. Only the magnitude of the voltage

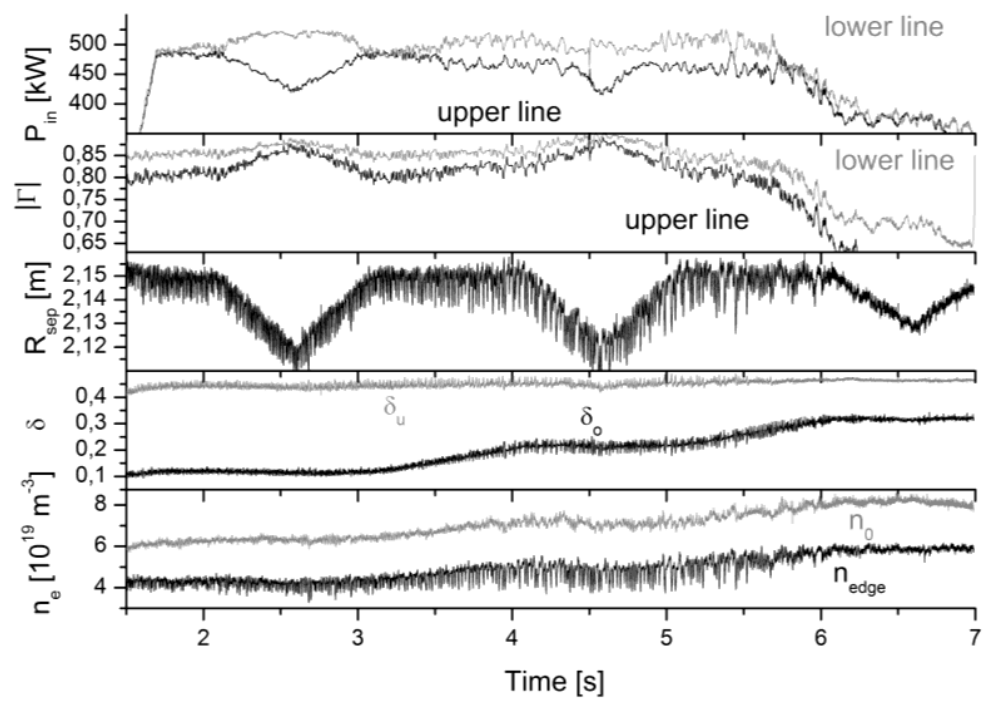

FIGURE 2. Time traces of the relevant signals during discharge 29823. $\mathrm{P}_{\text {in }}-$ coupled power, $\Gamma$ - voltage reflection coefficient (only antenna 1 for clarity),

$\mathrm{R}_{\text {sep }}-$ separatrix position (major radius), $\delta$ - triangularity, $\mathrm{n}_{\mathrm{e}}-$ electron density (edge and core, measured by DCN interferometer). The $|\Gamma|$ and $\mathrm{P}_{\text {in }}$ signals have been smoothed for clarity. 
reflection coefficient $\Gamma$ is plotted for brevity, although both magnitude and phase were measured. Between $2-3$ s and $4-5$ $|\Gamma|$ increases by about $5 \%$ on both lines due to $R_{\text {sep }}$ moving away from the antenna; there is also a slight increase between 3 and $4 \mathrm{~s}$, otherwise no obvious effect of $\delta_{\mathrm{o}}$ can be seen until $\sim 5.7 \mathrm{~s}$. At this point $\delta_{\mathrm{o}} \approx 0.29$ and high frequency type II ELMs appear, and $|\Gamma|$ decreases significantly on both lines, from $\sim 0.83$ (upper line) and $\sim 0.86$ (lower line) for $\mathrm{t}<5.7 \mathrm{~s}$ down to $\sim 0.55$ (upper) and $\sim 0.66$ (lower) at $\mathrm{t}=6.9 \mathrm{~s}$ (where the plasma is close to a double null configuration). The phase of $\Gamma$ underwent variations of $\sim 5^{\circ}$ during the same time; unlike the changes in $|\Gamma|$, however, these shifts were not larger than those observed during $R_{\text {sep }}$ scans. Note that the coupled power decreases significantly after $5.7 \mathrm{~s}$, since the antenna $Z_{\text {inp }}$ shifts out of the matching region. Similar effects were seen



FIGURE 3. $|\Gamma|$ as a function of $\delta_{\mathrm{o}}$, measured in the two $2.5 \mathrm{~T}$ discharges (29822 and 29823), and corresponding FELICE results. on all antennas during all shots.

Fig. 3 shows $|\Gamma|$ as a function of $\delta_{\mathrm{o}}$ between 5 and $7 \mathrm{~s}$; the change at $\delta_{\mathrm{o}} \approx 0.29$ is notable. This is a significant change in coupling, corresponding to an increase of roughly $124 \%$ (upper line) and $117 \%$ (lower line) in strap loading resistance with respect to the value at $\mathrm{t}<5.7 \mathrm{~s}$.

Furthermore, $|\Gamma|$ was also computed with FELICE (Finite Element Ion Cyclotron Emulator), an RF coupling/absorption code using a multispecies slab plasma model which includes finite Larmor radius effects. The antenna was modeled by two infinitely thin radiating straps with a perfect Faraday screen, having a forced current distribution and a phase difference of $\pi$ between them. In each case the density profile used as input was an average over $0.05 \mathrm{~s}$. A qualitative agreement can be seen, despite the large fluctuations in the measured profiles due to strong ELM activity.

\section{Effect of Magnetic Perturbations}

Four discharges were dedicated to this study; two at $B_{0}=2.5 \mathrm{~T}$ and two at $B_{0}=2 \mathrm{~T}$ (of which one was terminated early); coupled power was between 3.1 and 3.8 MW with all four antennas powered simultaneously between 1.5 and $8 \mathrm{~s}$. During the flat-top phase, central density was $n_{\mathrm{e} 0} \approx 8.5-9.5 \times 10^{19}$ $\mathrm{m}^{-3}$. MP coils were switched on during four $0.55 \mathrm{~s}$ intervals with $1.009 \mathrm{kA}$; the phase of the field was shifted by $\pi / 2$ in each interval. $R_{\text {sep }}$ scans were done during and between MPs. Fig. 4 shows the relevant time traces for discharge $29851\left(B_{0}=2.5 \mathrm{~T}\right)$. Type I ELMs are clearly seen between MPs, and are largely suppressed when the coils are on.

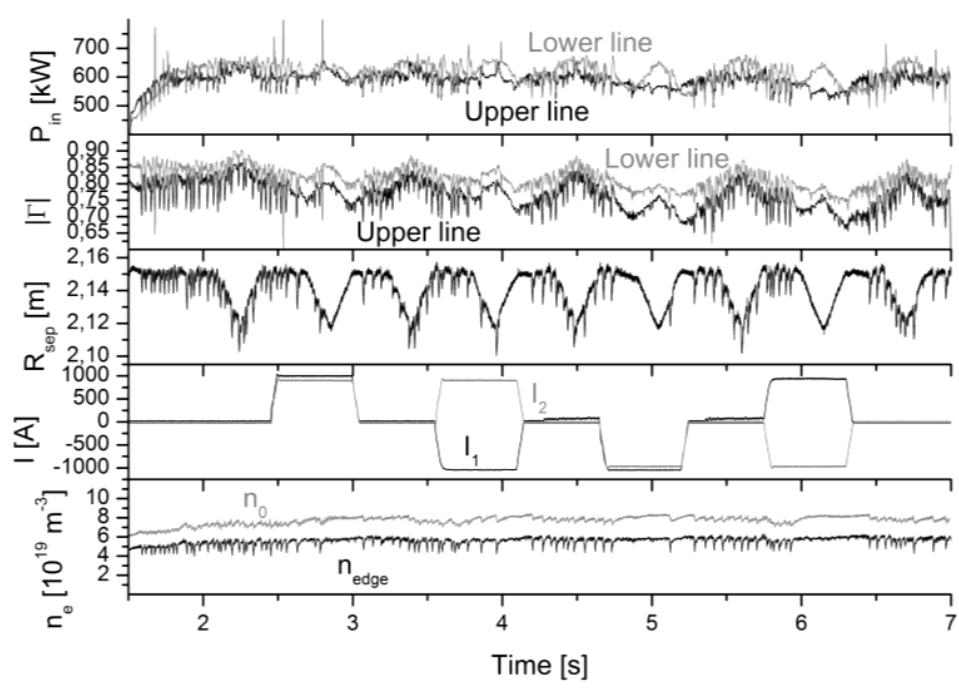

FIGURE 4. Time traces of the relevant signals during discharge 29851. $\mathrm{P}_{\text {in }}-$ coupled power, $\Gamma$ - voltage reflection coefficient (only antenna 1 for clarity), $\mathrm{R}_{\text {sep }}$ - separatrix position (major radius), $\mathrm{I}-$ MP coil current, $\mathrm{n}_{\mathrm{e}}$ - electron density (edge and core, measured by DCN interferometer). The $|\Gamma|$ and $\mathrm{P}_{\text {in }}$ signals have been smoothed for clarity. 
The effect of the MPs on coupling is shown in Fig. 5. It can be seen that $|\Gamma|$ is $7 \%$ (upper line) and 6\% (lower line) lower during MPs; from the averages of these datasets one computes an increase of roughly $30 \%$ (upper line) and $35 \%$ (lower line) in radiation resistance with respect to the value between MPs. The datasets shown are from discharge 29851; similar results were seen during the remaining shots. Although the large variations due to ELMs have been filtered out, $|\Gamma|$ still shows a large vertical spread due to the presence of high-frequency ELM-like events between ELMs, substantially more difficult to eliminate. It also must be added that changes in the phase of $\Gamma$ were in the range of $\sim 5^{\circ}$ during all the $R_{\text {sep }}$ scans, independently of whether the MP coils were switched on or not and their phasing.

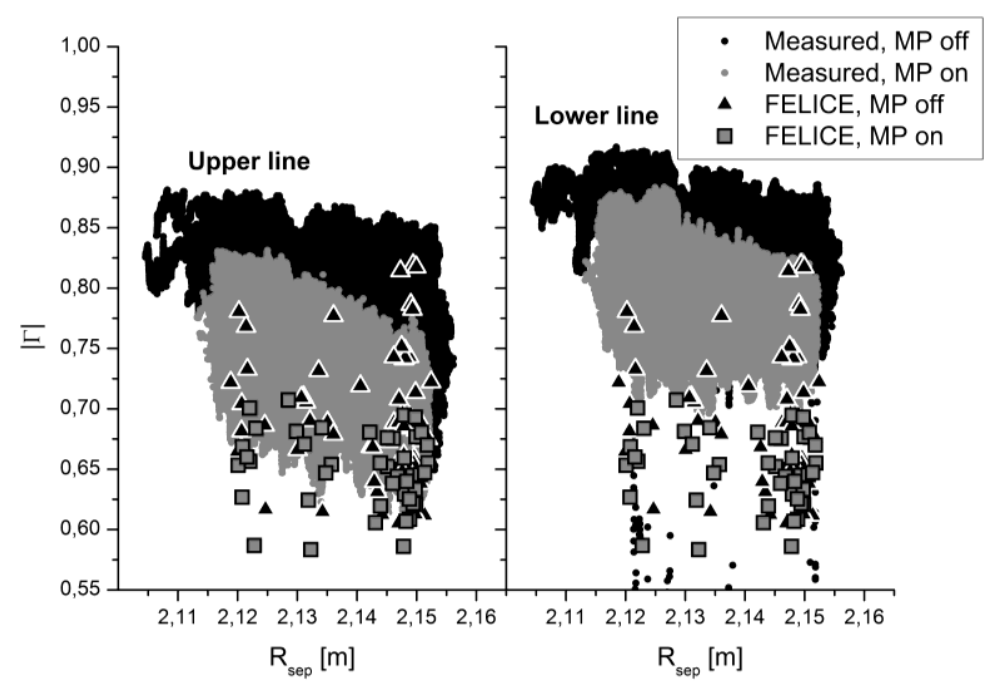

FIGURE 5. $|\Gamma|$ as a function of $R_{\text {sep }}$ on both lines, during and between MPs, and corresponding FELICE results.

The results of FELICE simulations are also shown. The input profiles were averaged over $0.05 \mathrm{~s}$. Again, the vertical spread on the results is rather large due to the ELM-like events, but the main trend is clearly visible in that, on average, the computed $|\Gamma|$ is lower when the MP coils are switched on. No obvious dependence on the phasing of the MP fields was observed.

\section{CONCLUSIONS AND REMARKS}

The effects of plasma triangularity and MPs on antenna reflection coefficients (equivalently, input impedance) have been studied on ASDEX Upgrade. New diagnostics, consisting of voltage and current probe pairs located close to the antenna input ports, complement the already existing measurements with DCs and yield additional information on the behavior of each antenna strap. It has been observed during the experiments that both parameters - triangularity and MPs - can markedly improve antenna coupling; specifically, decreases in $|\Gamma|$ of up to $~ 30 \%$ were observed during the triangularity scans and up to $\sim 7 \%$ during MPs. Simulation results are in qualitative agreement with these observations, despite the fact that ELM and ELM-like activity complicate the analysis somewhat. Further work will be directed towards studying the changes in the density profile due to both parameters, and simulations using a more sophisticated antenna model - with the coupling code TOPICA [5] - in order to obtain predictions not only about the magnitude of $\Gamma$ but also its phase.

\section{REFERENCES}

1. P. Jacquet et al., Nucl. Fusion 52, 042002 (2012).

2. V. Bobkov et al., Nucl. Fusion 46, S469-S475 (2006).

3. R. Fischer et al., Plasma Phys. Control. Fusion 54115008 (2012).

4. V. Bobkov et al., this conference.

5. V. Lancellotti et al., Nucl. Fusion 46 S476-S499 (2006).

6. I. Stepanov et al., Fusion Eng. Des. (2013), http://dx.doi.org/10.1016/j.fusengdes.2013.02.011

7. H. Faugel et al., this conference. 